\title{
A Survey: Digital Image Watermarking Techniques
}

\author{
Preeti Parashar ${ }^{1}$ and Rajeev Kumar Singh ${ }^{2}$ \\ PG Scholar ${ }^{1}$, Assistant Professor ${ }^{2}$ \\ Department of CSE \& IT, MITS, Gwalior, India \\ preeti.parashar@yahoo.co.in ${ }^{1}$,rajeev.mits1@gmail.com ${ }^{2}$
}

\begin{abstract}
Multimedia security is extremely significant concern for the internet technology because of the ease of the duplication, distribution and manipulation of the multimedia data. The digital watermarking is a field of information hiding which hide the crucial information in the original data for protection illegal duplication and distribution of multimedia data. This paper presents a survey on the existing digital image watermarking techniques. The results of various digital image watermarking techniques have been compared on the basis of outputs. In the digital watermarking the secret information are implanted into the original data for protecting the ownership rights of the multimedia data. The image watermarking techniques may divide on the basis of domain like spatial domain or transform domain or on the basis of wavelets. The spatial domain techniques directly work on the pixels and the frequency domain works on the transform coefficients of the image. This survey elaborates the most important methods of spatial domain and transform domain and focuses the merits and demerits of these techniques.
\end{abstract}

Keywords: Digital watermarking, Spatial domain, Least Significant Bit (LSB), Frequency domain, Discrete Cosine Transform (DCT), Discrete Wavelet Transform (DWT), Discrete Fourier Transform (DFT)

\section{Introduction}

Digital image processing is a rapidly developing area with various raising applications in computer science and engineering. It is very important field for the research work because its techniques are used in almost all kinds of tasks like human computer interface, medical visualisation; image enhancement, Law enforcement, artistic effects, image restoration and digital watermarking for security purpose. Digital image processing has many beneficial properties over the analogue image processing. Digital image processing is accomplishing variant computer operations on digital image for various purposes like enhancing image quality, filtering images from noise. A digital image [1] is a representation of two dimensional images as a finite set of digital values called picture elements or pixels. Therefore, processing a digital image by using a digital computer is called digital image processing.

The digital communication technology, like internet technology confronts various troubles related to the privacy and security of the data. Security techniques are required because of illegal access of data without permission. Therefore, it is necessary to protect data in the internet technology. For providing the security of digital data various techniques are used like encryption, decryption, cryptography, steganography and digital watermarking. In this paper discusses about the digital watermarking. The digital watermarking is an application of the digital image processing. 
The digital watermarking is a process of information hiding. There are various techniques for hiding the information in the form of digital contents like image, text, audio and video. Basically digital watermarking is a method for embedding some secret information and additional information in the cover image which can later be extracted or detected for various purposes like authentication, owner identification, content protection and copyright protection, etc. Sometimes the scaling factor is also used for embedding the watermark in the cover image. The digital watermarking is used for the security of the digital content and to protect the data from illegal users and provides the ownership right for the digital data. An important characteristic of digital watermarking is robustness and imperceptibility against various types of attacks or common image manipulation like rotation, filtering, scaling, cropping and compression. The efficiency of digital watermarking algorithms is totally based on the robustness of the embedded watermark against various types of attacks. Digital watermarking is a method used to improve the ownership over image by replacing low level signal directly into image. Digital watermarking method is also used for the tamper proofing and authentication [2].

Digital watermarking is a very developing field and used in various applications which have been proved to be successful. The digital watermarking has been applied in a number of image processing techniques. The aim of every application is to providing security of the digital content. The digital watermarking applications are Broadcast Monitoring [3], Digital Fingerprinting [4], Transaction Tracking [5], Copyright protection [6], Temper Detection [7], Data Hiding [8] and Content Authentication [9] etc.

Every digital watermarking technique includes two algorithms: one as the embedding algorithm and other as the detecting algorithm. These two processes are same for all the type of watermarking techniques. Figure 1 shows the watermark embedding process in which the watermark is embedded in the cover image by using the embedding algorithm. And Figure 2 shows the watermark detection process in which the embedded watermark is recovered by using the detection algorithm.

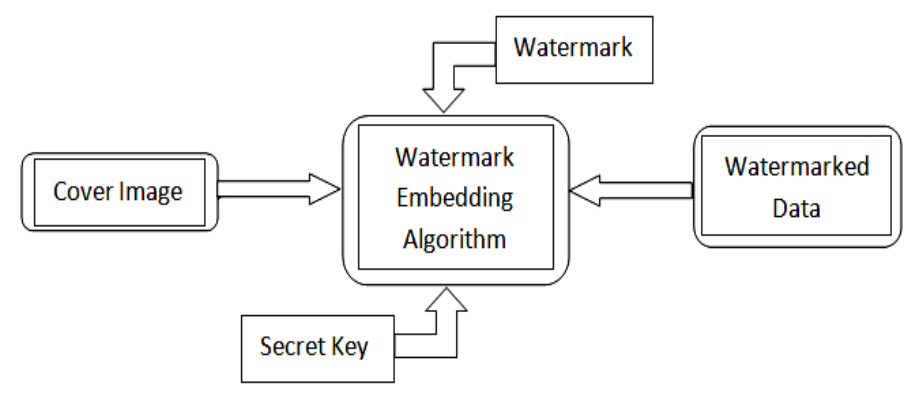

Figure 1. Watermark Embedding Process

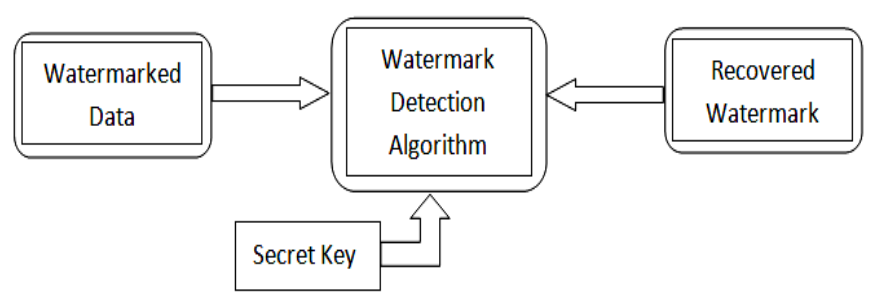

Figure 2. Watermark Detection Process 
This paper is categorized in different sections. In Section 2, we have discussed working of digital image watermarking with the stages explanation. Section 3 defines digital watermarking techniques and Section 4 describes the experimental results of some important spatial domain or transforms domain techniques. Finally, Section 5 draws conclusions.

\section{Digital Image Watermarking Working}

Digital Watermarking is a technique which is used in the digital signal processing of embedding hidden information into multimedia data. This information is not usually visible, only dedicated detector or extractor can seen and extracts that information. Digital Image Watermarking use digital image for embedding the hidden information, after embedding the watermarked image is generated and the watermarked image is more robust against attacks.

Figure 3 shows the stages of digital watermarking. Basically working of digital image watermarking can be divided in three stages [10]:

\subsection{Embedding Stage}

The embedding stage is the first stage in which the watermark is embedded in the original image by using the embedding algorithm and the secret key. Then the watermarked image is generated. So the watermarked image is transmitted over the network.

\subsection{Distortion/Attack Stage}

In this stage, when the data is transmitted over the network. Either some noise is added with the watermarked image or some attacks are performed on the watermarked image. So, our watermarked data is either modified or destroyed.

\subsection{Detection/Retrieval Stage}

In the detection stage, the watermark is detected or extracted by the dedicated detector from the watermarked image by applying some detection algorithm and by using secret key. In addition to this, noise is also detected.

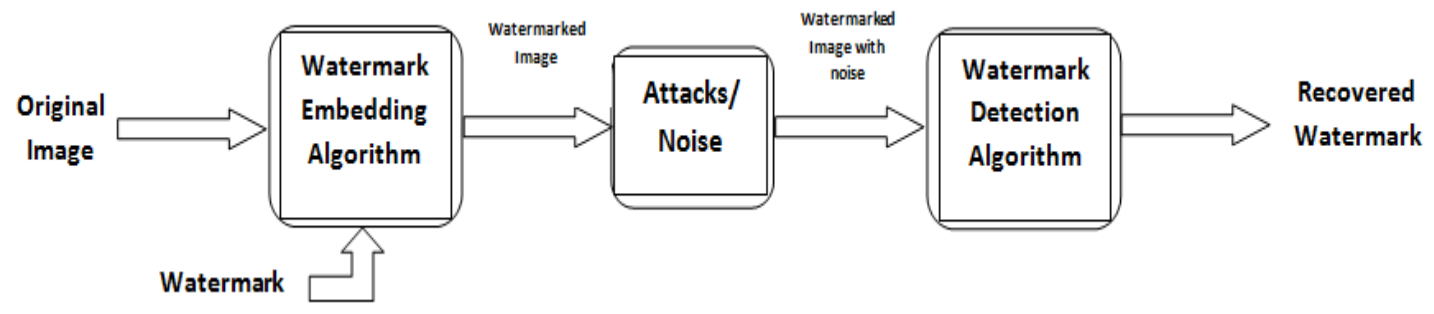

Figure 3. Stages in Digital Image Watermarking

\section{Digital Image Watermarking Techniques}

In the field of digital watermarking, digital image watermarking has attracted a lot of awareness in the research community for two reasons: one is its easy availability and the other is it convey enough redundant information that could be used to embed watermarks [11]. Digital watermarking contains various techniques for protecting the digital content. The entire digital image watermarking techniques always works in two domains either spatial domain or transform domain. The spatial domain techniques works directly on pixels. It embeds the watermark by modifying the pixels value. Most commonly used spatial domain techniques are LSB. Transform domain techniques embed the watermark by modifying the transform domain coefficients. Most commonly used transform domain techniques is DCT, 
DWT and DFT. For achieving the robustness and imperceptibility, the transform domain techniques are more effective as compare to the spatial domain. We further elaborated these two domains and its techniques.

\subsection{Spatial Domain Watermarking}

The spatial domain represents the image in the form of pixels. The spatial domain watermarking embeds the watermark by modifying the intensity and the colour value of some selected pixels [12]. The strength of the spatial domain watermarking is

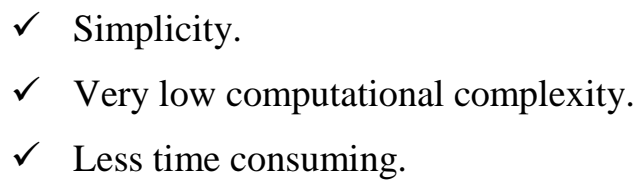

The spatial domain watermarking is easier and its computing speed is high than transform domain but it is less robust against attacks. The spatial domain techniques can be easily applied to any image. The most important method of spatial domain is LSB.

\section{Least Significant Bit (LSB):}

The LSB is the simplest spatial domain watermarking technique to embed a watermark in the least significant bits of some randomly selected pixels of the cover image. Example of least significant bit watermarking [12]:

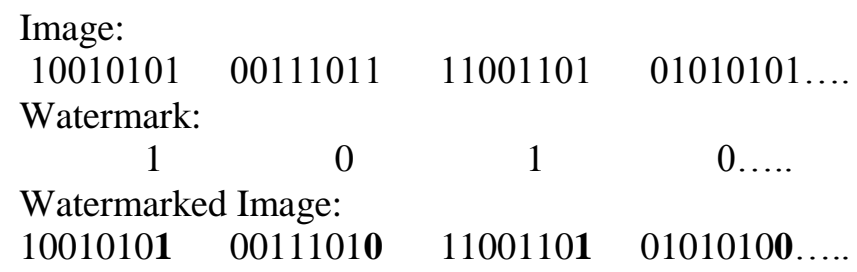

The steps used to embed the watermark in the original image by using the LSB [13]:

1) Convert RGB image to grey scale image.

2) Make double precision for image.

3) Shift most significant bits to low significant bits of watermark image.

4) Make least significant bits of host image zero.

5) Add shifted version (step 3) of watermarked image to modified (step 4) host image.

The main advantage of this method is that it is easily performed on images. And it provides high perceptual transparency. When we embed the watermark by using LSB the quality of the image will not degrade. The main drawback of LSB technique is its poor robustness to common signal processing operations because by using this technique watermark can easily be destroyed by any signal processing attacks. It is not vulnerable to attacks and noise but it is very much imperceptible. 


\section{Limitations of spatial domain watermarking:}

The spatial domain watermarking is simple as compared to the transform domain watermarking. The robustness is the main limitation of the spatial domain watermarking. It can survive simple operations like cropping and addition of noise.

Another limitation of spatial domain technique is that they do not allow for the subsequent processing in order to increase the robustness of watermark.

\subsection{Transform Domain Watermarking}

The transform domain watermarking is achieving very much success as compared to the spatial domain watermarking. In the transform domain watermarking, the image is represented in the form of frequency. In the transform domain watermarking techniques, firstly the original image is converted by a predefined transformation. Then the watermark is embedded in the transform image or in the transformation coefficients. Finally, the inverse transform is performed to obtain the watermarked image [14]. Most commonly used transform domain methods is Discrete Cosine Transform (DCT), Discrete Wavelet Transform (DWT) and Discrete Fourier Transform (DFT).

3.2.1. Discrete Cosine Transform: Discrete Cosine Transform (DCT) used for the signal processing. It transforms a signal from the spatial domain to the frequency domain. DCT is applied in many fields like data compression, pattern recognition and every field of image processing. DCT watermarking is more robust as compared to the spatial domain watermarking techniques. The main steps which used in DCT [11]:

1) Segment the image into non-overlapping blocks of $8 x 8$.

2) Apply forward DCT to each of these blocks.

3) Apply some block selection criteria (e.g. HVS).

4) Apply coefficient selection criteria (e.g. highest).

5) Embedded watermark by modifying the selected Co-efficient.

6) Apply inverse DCT transform on each block.

In DCT, for embedding the watermark information, we divide the image into different frequency bands. In Figure 4 FL denotes the lowest frequency component of the block, while FH denotes the higher frequency component and FM denotes the middle frequency component which is chosen as the embedding region. The Discrete cosine transform achieves good robustness against various signal processing attacks because of the selection of perceptually significant frequency domain coefficients.

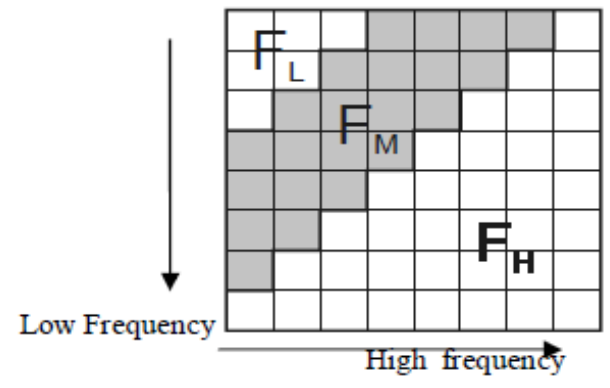

Figure 4. Discrete Cosine Transform Region 
The most common DCT definition of a 1-D sequence of length $\mathrm{N}$ is [15]:

$$
c(u)=\alpha(u) \sum_{x=0}^{N-1} f(x) \cos \left[\frac{(2 x+1) u \pi}{2 N}\right]
$$

For $\mathrm{u}=0,1,2,3 \ldots . \mathrm{N}$-1.Similarly, the inverse transformation for 1-D sequence of length $\mathrm{N}$ is

$$
f(x)=\sum_{u=0}^{N-1} \alpha(u) c(u) \cos \left[\frac{(2 x+1) u \pi}{2 N}\right]
$$

For both the equations $\alpha(\mathrm{u})$ is defined as

$$
\alpha(u)= \begin{cases}\sqrt{\frac{1}{N}}, & u=0 \\ \sqrt{\frac{2}{N}}, & u \neq 0\end{cases}
$$

In DCT the first transform coefficient is DC coefficient and all others are AC coefficients. The 2-D DCT transform is extension of 1-D DCT and is given by [16]:

$$
c(u, v)=\alpha(v) \alpha(u) \sum_{x, y=0}^{N-1} \sum f(x, y) \cos \left[\frac{(2 x+1) u \pi}{2 N}\right] \cos \left[\frac{(2 y+1) v \pi}{2 N}\right]
$$

For $\mathrm{u}, \mathrm{v}=0,1,2,3 \ldots . ., \mathrm{N}-1$ and $\alpha(\mathrm{u})$ and $\alpha(\mathrm{v})$ defined in equation (3). The 2-D DCT inverse transforms is given by:

$$
f(x, y)=\sum_{u, v=0}^{N-1} \sum \alpha(v) \alpha(u) c(u, v) \cos \left[\frac{(2 x+1) u \pi}{2 N}\right] \cos \left[\frac{(2 y+1) v \pi}{2 N}\right]
$$

Merits of DCT:

$>\mathrm{DCT}$ is better than any of the spatial domain techniques because it is robust against varies kinds of attacks like cropping, noising, filtering and sharpening.

$>$ DCT is a real transform with better computational efficiency.

$>$ The DCT gives a better performance in the bit rate reduction.

$>$ DCT also implement fast algorithms.

3.2.2. Discrete Wavelet Transform: Discrete wavelet transform (DWT) of the image produces multi resolution representation of an image. The multi resolution representation provides a simple framework for interpreting the image information. The DWT analyses the signal at multiple resolution. DWT divides the image into high frequency quadrants and low frequency quadrants. The low frequency quadrant is again split into two more parts of high and low frequencies and this process is repeated until the signal has been entirely decomposed. 
The single DWT transformed two dimensional image into four parts: one part is the low frequency of the original image, the top right contains horizontal details of the image, the one bottom left contains vertical details of the original image, the bottom right contains high frequency of the original image. The low frequency coefficients are more robust to embed watermark because it contains more information of the original image [2]. The reconstruct of the original image from the decomposed image is performed by IDWT [16].

The digital wavelet transform are scalable in nature. DWT more frequently used in digital image watermarking because of its excellent spatial localization and multi resolution techniques. The excellent spatial localization property is very convenient to recognize the area in the cover image in which the watermark is embedded efficiently.

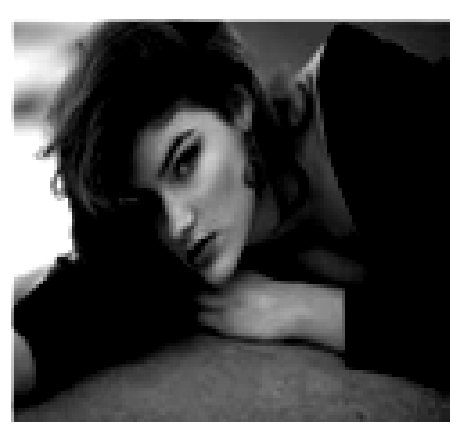

(a) Host Image

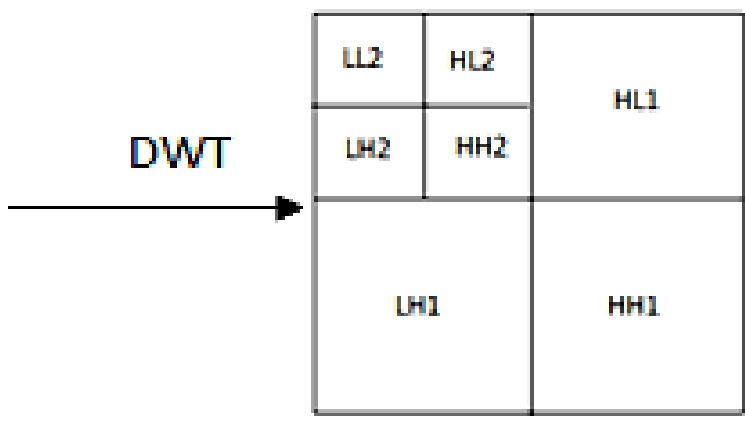

b) DWT Transform

Figure 5. Two Level Decomposition

The DWT is applied on the host image to decompose the image into four non overlapping multi resolution coefficient sets. The coefficients are [28]:

$$
\begin{aligned}
W_{L L}^{J} & =\sum_{x=0}^{N-1} \sum_{y=0}^{N-1} g(x) g(y) W_{L L}^{J-1}(2 u-x)(2 v-y) \\
W_{L H}^{J} & =\sum_{x=0}^{N-1} \sum_{y=0}^{N-1} g(x) h(y) W_{L L}^{J-1}(2 u-x)(2 v-y) \\
W_{H L}^{J} & =\sum_{x=0}^{N-1} \sum_{y=0}^{N-1} h(x) g(y) W_{L L}^{J-1}(2 u-x)(2 v-y) \\
W_{H H}^{J} & =\sum_{x=0}^{N-1} \sum_{y=0}^{N-1} h(x) h(y) W_{L L}^{J-1}(2 u-x)(2 v-y)
\end{aligned}
$$

Where $\mathrm{J}$ is the level of the 2-D DWT, $\mathrm{h}(\mathrm{n})$ and $\mathrm{g}(\mathrm{n})$ are the impulse response. Figure 6 shows the schematic diagram of $2 \mathrm{D}$ wavelet transform. By using this figure we can analyse in which way the $2 \mathrm{D}$ wavelet transform are performed. 


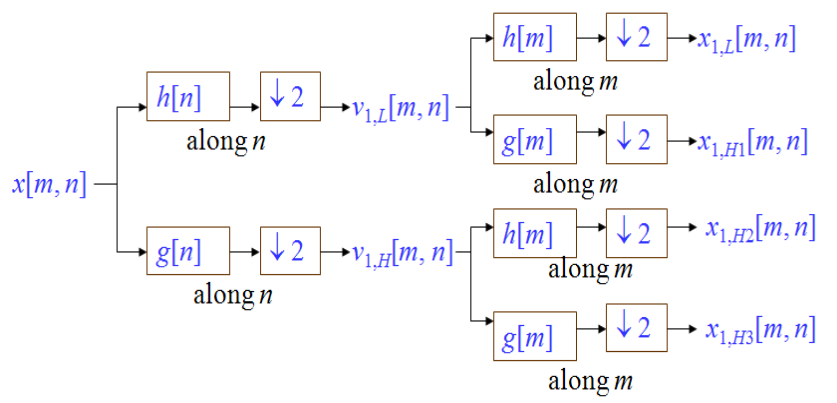

Figure 6. Schematic Diagram of 2D Wavelet Transform [19]

\section{Merits of DWT over DCT:}

$>$ DWT gives better visual image quality as compared to the DCT.

$>$ In DWT, dividing the input coding into non overlapping 2-D block is not necessary; its higher compression ratios avoid blocking artefacts.

$>$ DWT allows better localization as compared to the DCT.

$>$ The watermarking method is robust to wavelet transform based image compression as well as to other common image distortions like rescaling half toning, additive noise etc. This is also an advantage over DCT [11].

$>$ The DWT understands the working of HVS more clearly than the DCT.

$>$ DWT defines the multi resolution description of the image. So, the image can be shown in different levels of resolution and proceed from low resolution to high resolution.

\section{Demerits of DWT over DCT:}

The main disadvantage of DWT is that the DWT is more complex than the DCT. When DCT is used it takes 54 multiplications to compute for a block of $8 \times 8$, distinct wavelet calculation depends upon the length of the filter used, whom at least one multiplication per coefficient. The other drawback is that computation cost is higher and its computation time is longer.

3.2.3. Discrete Fourier Transform: Discrete Fourier Transform (DFT) offers robustness against geometric attacks like rotation, scaling, cropping, translation etc. DFT decomposes an image in sine and cosine form. The DFT based watermark embedding techniques are divided in two types: one is the direct embedding and the other one is the template based embedding.

According to the direct embedding technique the watermark is embedded by modifying DFT magnitude and phase coefficients. The template based embedding technique introduces the concept of templates. A template is structure which is embedded in the DFT domain to estimate the transformation factor. Once the image undergoes a transformation this template is searched to resynchronize the image, and then the detector is used to extract the embedded spread spectrum watermark [11].

The DFT is used for the periodic, digital signals or discrete-time $\mathrm{f}(\mathrm{x})$. The DFT for a signal with period $\mathrm{M}$ is [1]: 


$$
F(u)=\sum_{x=0}^{M-1} f(x) e^{-\frac{j 2 \pi u x}{M}}
$$

The Inverse Discrete Fourier Transform (IDFT) is:

$$
f(x)=\frac{1}{M} \sum_{u=0}^{M-1} F(u) e^{-\frac{j 2 \pi u x}{M}}
$$

Where, $\mathrm{u}, \mathrm{x}=0,1,2 \ldots, \mathrm{M}-1$. Equations (13) and (14) are the pair of 1-D Discrete Fourier Transform. Both the forward and inverse discrete transform are infinitely periodic, with period M. i.e.

And

$$
F(u)=F(u+k M)
$$

$$
f(x)=f(x+k M)
$$

\section{Some characteristics of DFT:}

In the DFT, real image is normally complex valued, which results in the phase and magnitude representation of an image.

The main \& strongest component of the DFT is the central component which contains low frequency.

DFT is also resistant to cropping because effect of cropping leads to the blurring of spectrum. If the watermarks are embedded in the magnitude, which are normalized coordinates, there is no need of any synchronization.

Scaling of image results in amplification of extracted signal and can be detected by correlation coefficient.

\section{Advantages of DFT over DWT and DCT:}

The DFT is Rotation Scaling Translation (RST) invariant. So, DFT can be used to recover from geometric distortion, whereas the spatial domain, DCT and DWT are not RST invariant. Hence, it is difficult to overcome from geometric distortions [11].

\section{Disadvantage of DFT over DWT and DCT:}

The main disadvantage of the DFT is that the output of the DFT is always in complex value and it requires more frequency rate. Its computational efficiency is very poor. So, the DFT not used because of these disadvantages.

\section{Experimental Results}

This section elaborates the experimental results of digital image watermarking techniques in MATLAB. Digital image watermarking techniques works in two domains: spatial domain and transform domain. The results of the most important methods of spatial domain as well as transform domain are explained below.

In the experimental results, firstly a GUI for showing the results effectively and implement the most important methods of the spatial domain and transform domain is created. The 
methods are evaluated on a sample image; either grayscale or RGB image of any size and then watermark image is embedded in the original selected image. After performing LSB method of the spatial domain, the watermarked image is generated. In LSB, the watermark image is embedded into the least significant bits of original image. Mostly used transform domain methods are DCT, DWT and DFT which are used in many fields like compression pattern recognition and in every field of image processing. Then a method of transform domain is applied and transformed image is generated.

Figure 6(a) shows the GUI of digital image watermarking techniques. (b) The original image which is selected (c) the selected watermark image. (d) Watermarked image after embedding the watermark using LSB method of spatial domain. (e) Shows the DWT transform of original image. The DWT transform the image into multiple resolutions. (f) Shows the DCT transform of the image. The DCT transform the image into different frequency bands. (g) Shows the DFT transform of original image. The DFT transform the image in sine and cosine form.

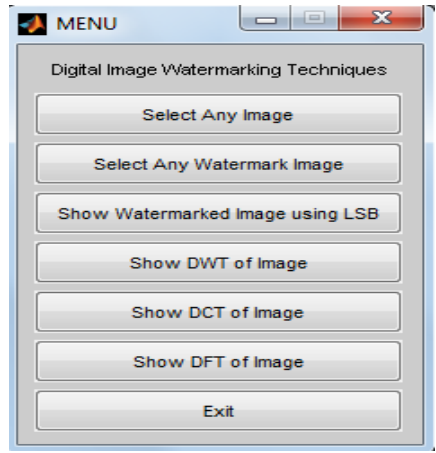

\section{a) GUI of Digital Image Watermarking Techniques}

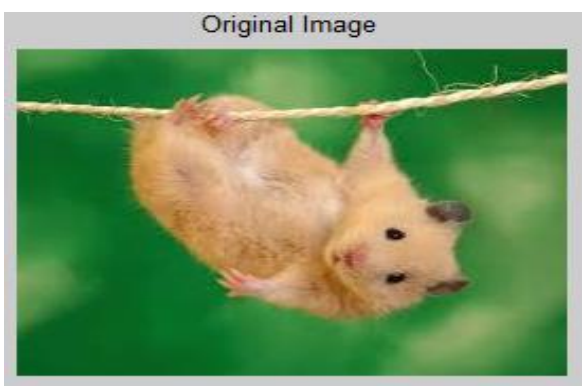

b) Original Image

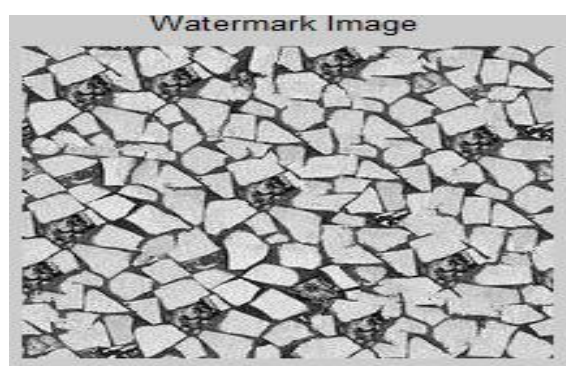

c) Watermark Image 


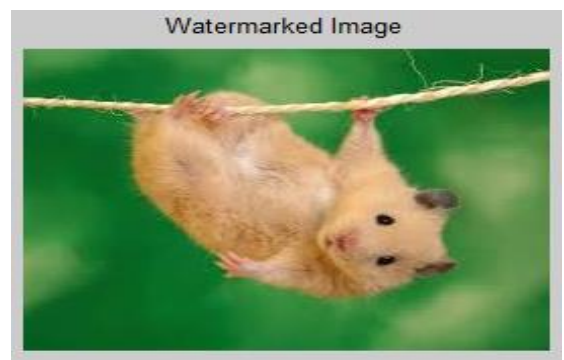

d) Watermarked Image using LSB

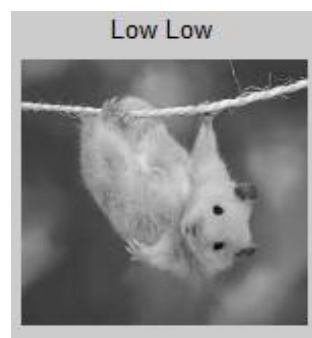

High Low

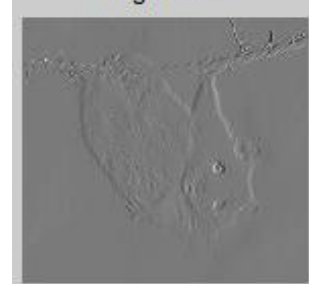

e) DWT of Original Image
High High
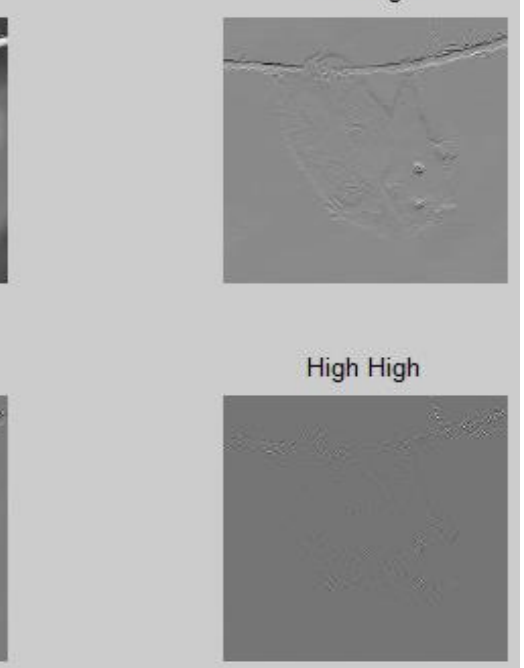

(1)

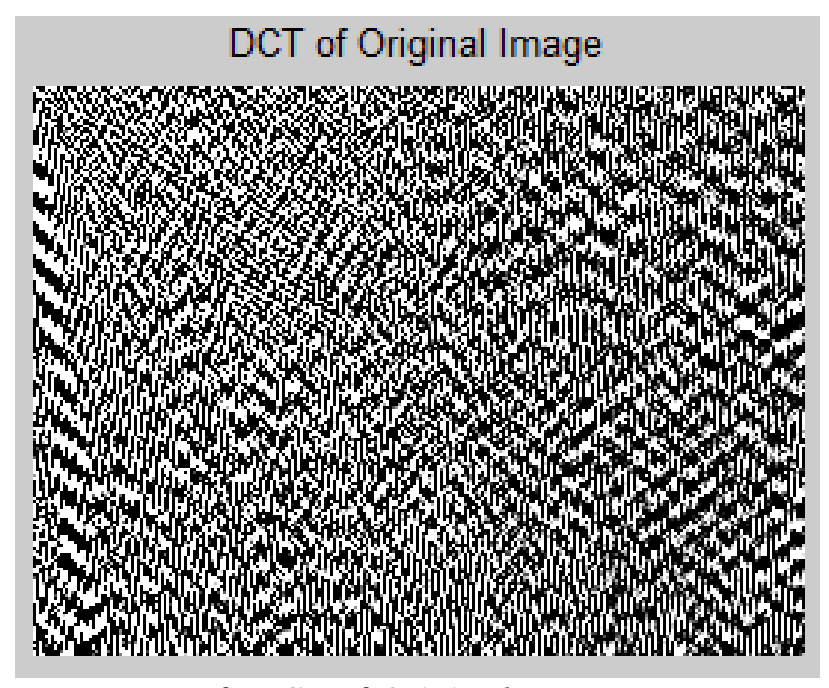

f) DCT of Original Image 


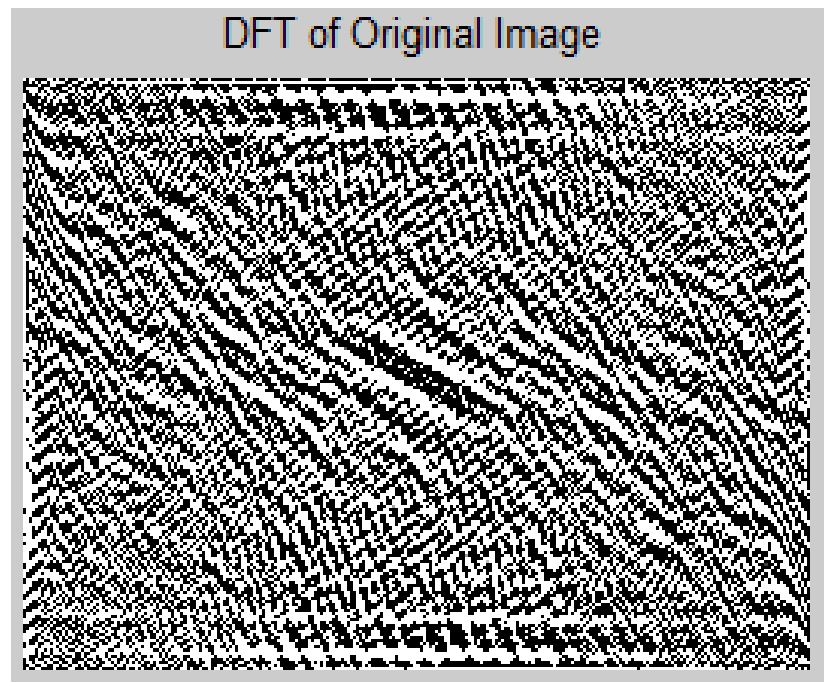

g) 1-DFT of Original Image

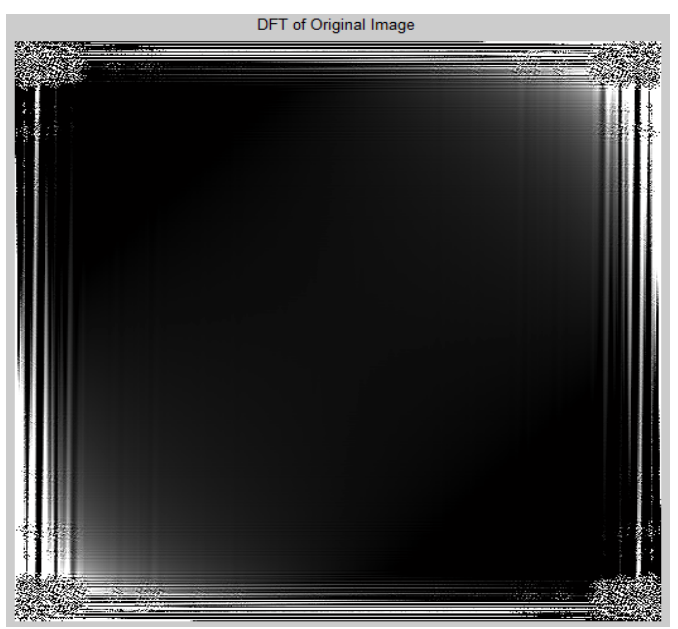

h) 2-DFT of Original Image

Figure 6. g shows the DFT of original image after converting it into greyscales. h) 2 show the DFT of original image after converting it into double

\section{Conclusion}

Digital watermarking is very useful method for providing security to the digital media on the internet technology. In this paper, survey of different techniques based on spatial domain (LSB) and the transform domain (DCT, DWT, DFT). This survey analyses the limitations and strengths of the watermarking methods.

Digital watermarking is still a challenging research field with many interesting problems, like it does not prevent copying or distribution and also cannot survive in every possible attack. One future research pointer is the development of truly robust, transparent and secure watermarking technique for different digital media including images, video and audio. 


\section{References}

[1] G. Rafael, C. Gonzalez and R. E. Woods, "Digital Image Processing", ThirdEdition, (2008).

[2] N. Tiwari, M. k. Ramaiya and Monika Sharma, "Digital watermarking using DWT and DES", IEEE (2013).

[3] L. Li and X. Li," Watermarking Protocol for Broadcast Monitoring", International Conference on E business and E-Government (ICEE) (2010).

[4] D. Zhang, S. Xu, Y. Wang, J. Zhang and Y. Li, "A Digital Fingerprinting Scheme of Digital Image". International Conference on Computational Intelligence and Software Engineerin (CISE) (2010).

[5] S. Emmanuel, A. P. Vinod, D. Rajan and C.K. Heng, "An Authentication Watermarking Scheme with Transaction Tracking Enabled”, Digital Ecosystem and Technologies Conference, 2007.DEST'07 Inaugural IEEE-IES.

[6] Y.-C. Wang and J.-f. Niu, "Research on Digital Content Copyright Protection System", IEEE International Conference on Network Infrastructure and Digital Content, 2009. IC-NIDC (2009).

[7] S.-L. Hsieh, C.-P. Yeh and I-J. Tsai, "An Image Copyright Protection Scheme with Tamper Detection Capability”, Symposia and Workshops on Ubiquitous, Autonomic and trusted Computing,2009.UIC-ATC'09

[8] Z. Ni, Y.Q. Shi, N. Ansari, W. Su, Q. Sun and X. Lin, "Robust Lossless Image Data Hiding Designed for Semi-Fragile Image Authentication”, IEEE Transactions on Circuits and Systems for Video Technology, vol. 18 , no. 4.

[9] J. Zhu, Q. Wei, J. Xiao and Y. Wang," A Fragile Software Watermarking Algorithm for Content Authentication", IEEE Youth Conference on Information, Computing and Telecommunication, 2009. YCICT'09.

[10] L. Robert and T. Shanmugapriya," A Study on Digital Watermarking Techniques", International Journal of Recent Trends in Engineering, vol. 1, no. 2, (2009) May.

[11] V. M. Potdar, S. Han and E. Chang, "A Survey of Digital Image Watermarking Techniques", $20053^{\text {rd }}$ IEEE International Conference on Industrial Informatics (INDIN).

[12] N. Chandrakar and J. Baggaa,"Performance Comparison of Digital Image Watermarking Techniques: A Survey", International Journal of computer Application Technology and Research, vol. 2, no. 2, (2013), pp. 126-130.

[13] D. Mistry," Comparison of Digital Watermarking Methods" (IJCSE) International Journal on Computer Science and Engineering, vol. 02, no. 09, (2010), pp. 2805-2909.

[14] F. Daraee and S. Mozaffari, "Watermarking in binary document images using fractal codes", Pattern Recognition Letter (2013).

[15] S. A. Khayam, "Discrete Cosine Transform (DCT): Theory and Application".

[16] S. S. Gonge and J. W. Bakal, "Robust Digital Watermarking Techniques by Using DCT and Spread Spectrum", International Journal of Electrical, Electronics and Data Communication, ISSN: 2320-2084, vol. 1, no. 2, (2013).

[17] B. Sridhar and C. Arun, "A Wavelet based Image Watermarking Technique using Image Sharing Method", IEEE (2013).

[18] K. Deb, Md. S. Al-Seraj, Md. M. Hoque and Md. I. H. Sarkar, "Combined DWT-DCT Based Digital Image Watermarking Technique for Copyright Protection”, International Conference on Electrical and Computer Engineering, (2012) December 20-22.

[19] C. -L. Liu, “A Tutorial of the Wavelet Transform”, (2010) February 23.

\section{Authors}

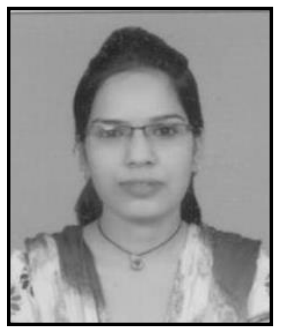

Preeti Parashar, she has received the BE degree in computer science and engineering form NRI-ITM, Gwalior, India 2011. Currently she is pursuing M.Tech in Computer Science and Engineering from MITS, Gwalior and it will be completed in 2014. Her research includes digital image processing and especially in digital watermarking. 
International Journal of Signal Processing, Image Processing and Pattern Recognition Vol. 7, No. 6 (2014)

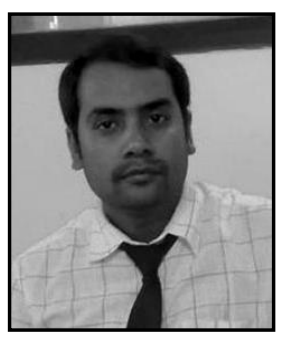

Rajeev Kumar Singh, he is an Assistant Professor, Department of Computer Science \& Information Technology, MITS, Gwalior, India. He has 3 years of teaching experience. His teaching and research include Digital Image Processing and Software Engineering. 